\title{
Hydrodynamic efficiency versus structural longevity of a fixed OWC wave energy converter
}

\author{
María L. Jalón ${ }^{\mathrm{a}, *}$, Feargal Brennan ${ }^{\mathrm{a}}$ \\ ${ }^{a}$ Department of Naval Architecture, Ocean \& Marine Engineering. University of Strathclyde. \\ Henry Dyer Building, 100 Montrose Street, Glasgow G4 OLZ, United Kingdom.
}

\begin{abstract}
This paper explores a trade-off between structural longevity and energetic response for wave energy converters in irregular waves. To this end, a physics-based simulation methodology is developed which integrates both the hydrodynamic efficiency calculation and fatigue damage assessment. This methodology is embedded into a parameterised computational model by coupling analytical models for the simulation of both the pressure field and the hydrodynamic efficiency for a particular incident sea state, and a numerical model for the structural analysis of the system. The methodology is general but for this paper it is specialised for an idealised power-optimal bottom-fixed oscillating water column device. As particular case studies, a sea-state adaptive power-optimal configuration and a fixed power-optimal configuration were investigated and compared. The results reveal that the sea-state adaptive configuration not only provides the optimal hydrodynamic efficiency, but also leads to less accumulated fatigue damage.
\end{abstract}

Keywords: Fatigue damage, hydrodynamic efficiency, irregular waves, oscillating water column, power-optimal configuration.

\section{Introduction}

The extraction of wave energy from the seas is a technology currently under development as an alternative to fossil fuels [1-4]. This technology is not yet competitive in terms of energetic efficiency [5], and an increasing literature tends to focus on investigating optimization strategies to maximise power extraction. To provide an overview, a number of authors have focused on finding power-optimal geometries for different wave energy converters (WECs) [6-9], while others have focused on maximizing the power take-off (PTO), which is the mechanism to transform the wave energy in electrical energy [10, 11]. A small number of studies have focused on optimizing both the geometry and the PTO like Falcão et al. [12] and Jalón et al. [13], applied to an oscillating water column (OWC) device.

However, apart from hydrodynamic efficiency considerations, WEC systems are subjected to progressive degradation due to the cyclic wave loading and the marine environment. This leads to operational downtime

*Corresponding author. e-mail: mljalon@ugr.es 
which effectively decreases the overall power efficiency of the WECs; therefore a few authors have recently started to investigate the structural integrity of these systems in the context of system design optimization. In particular, Tom et al. [14] formulated a power optimization problem for an oscillating-body WEC and evaluated the fatigue damage of the resulting power-optimal device using a spectral approach. A closedform expression was adopted to evaluate the cumulative fatigue damage by assuming that the peak stresses follow a Rayleigh distribution. Later, Tom et al. [15] extended their previous research to include the surge foundation force in the optimization problem such that the resulting design maximises the absorbed energy while minimizing structural loads; however, structural integrity was not assessed. A similar methodology was applied to an asymmetric heave WEC in regular and irregular waves in [16].

In this paper, a rigorous simulation methodology to investigate the trade-off between the energetic response and the structural longevity of WEC systems in irregular waves is developed. This methodology is embedded within a parameterised computational model by coupling analytical models solved in Python ${ }^{\circledR}[17]$ and a structural finite-element (FE) model developed in ABAQUS ${ }^{\circledR}$ [18], which integrates both the hydrodynamic efficiency calculation and the fatigue damage assessment. To this end, an irregular sea state is initially simulated by assuming that the free surface of the incident wave train behaving as an ergodic Gaussian stochastic process resulting from the superposition of monochromatic waves, which are characterised by assuming a particular energy density spectrum curve. Next, the hydrodynamic efficiency is computed using a spectral approach, while the associated pressure fields are calculated under a temporal approach. In particular, the associated pressure fields are obtained using the linear wave theory considering that the irregular sea state can be represented as a sequence of regular waves. The pressure fields are then introduced as time-series input loads to the structural finite-element model of the WEC, obtaining a timeseries of the maximum principal stresses at particular measurement points. Finally, the fatigue cycles and the corresponding stress ranges are obtained naturally by using the rainflow counting method, avoiding the assumption of a particular probability model for the stress ranges (e.g, Rayleigh). The accumulated fatigue damage is then estimated by using a suitable S-N curve using the Palmgren-Miner cumulative rule.

The proposed methodology is illustrated for an idealised bottom-fixed OWC system at a particular location considering two different system configurations, namely: (1) a sea state power-optimal configuration capable of automatically adopting the optimal configuration for a particular sea state, (2) a power-optimal annual configuration, which remains fixed along the time. The results show that the sea state power-optimal configuration leads to the best trade-off between hydrodynamic efficiency and structural longevity; i.e., the sea state power-optimal configuration not only leads to the optimal hydrodynamic efficiency, as previously shown by Jalón et al. in [13], but also to a more reliable system in terms of accumulated fatigue damage.

The remainder of the paper is organised as follows: Section 2 describes the methodology used to model the interaction of the irregular waves with the OWC system, the structural longevity, and the energetic response of the device. In Section 3, this methodology is applied for different optimal configurations developed by 
Jalón et al. [13], and the results are presented. Section 4 discusses the main results, and Section 5 lists the conclusions that can be derived from this research. 


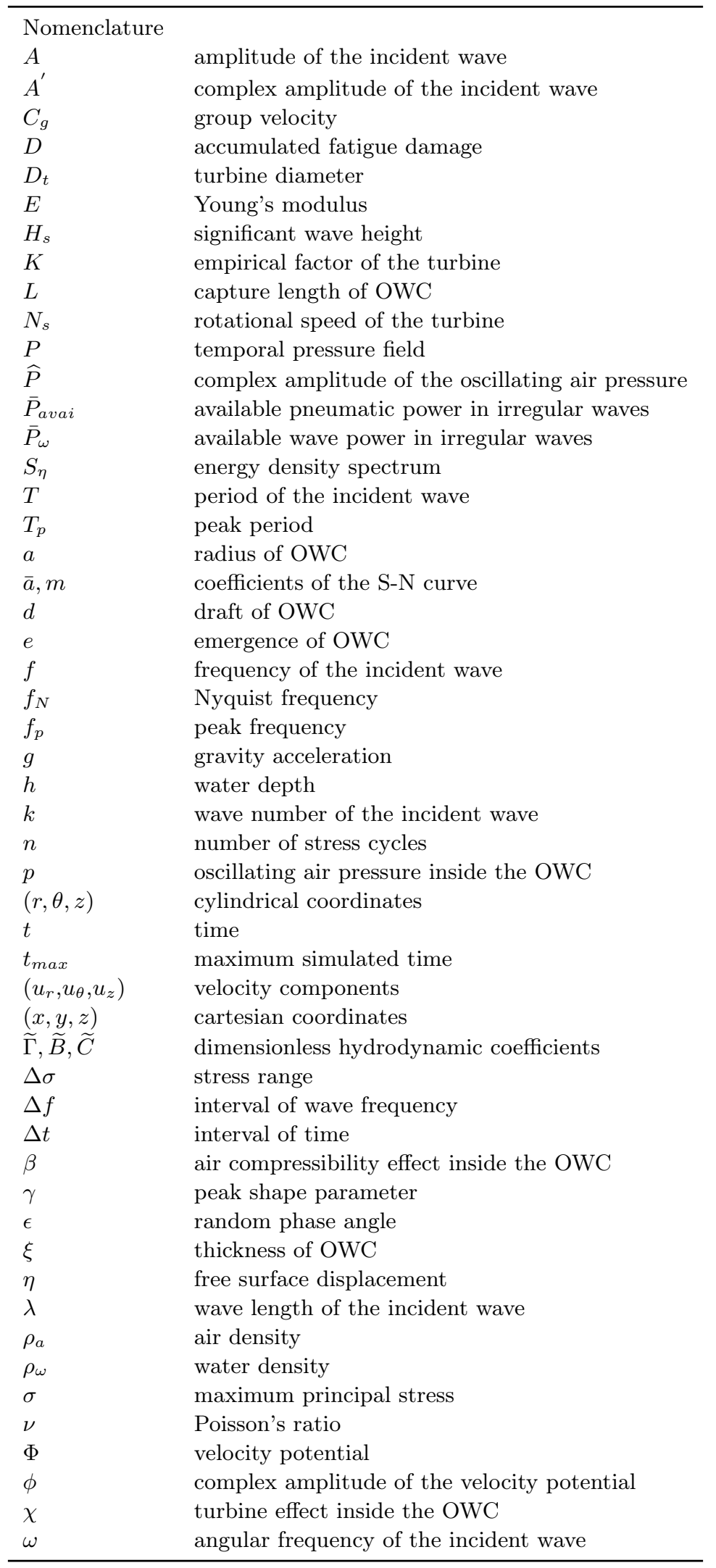




\section{Methodology}

For the purpose of system modelling, both the hydrodynamic efficiency and the accumulated fatigue damage are adopted as measures of the energetic response and the structural longevity, respectively. In this sense, a parameterised computational model which integrates both the hydrodynamic efficiency and the accumulated fatigue damage of a bottom-fixed OWC in irregular waves is developed to automate the simulation process. The simulation model is developed by coupling analytical models and a structural FE model, taking into account the environmental conditions of the study area and the description of the OWC system (material properties and configuration). An algorithmic description of the simulation tool is provided in Appendix A, and a flowchart outlining the different components of the model is given in Fig. 1.

As depicted in Fig. 1, the simulation of irregular waves is explained in Section 2.1 first. Next, the timedependent pressure field in irregular waves is calculated using Python ${ }^{\circledR}$ by applying an analytical model developed in Section 2.2.1. This time-dependent pressure field is applied as an input load to the structural FE model of the OWC system developed in ABAQUS ${ }^{\circledR}$, where the stresses are measured and the accumulated fatigue damage is calculated in Section 2.2.2. Finally, the hydrodynamic efficiency is calculated in Python ${ }^{\circledR}$ using the analytical model developed in Section 2.3.

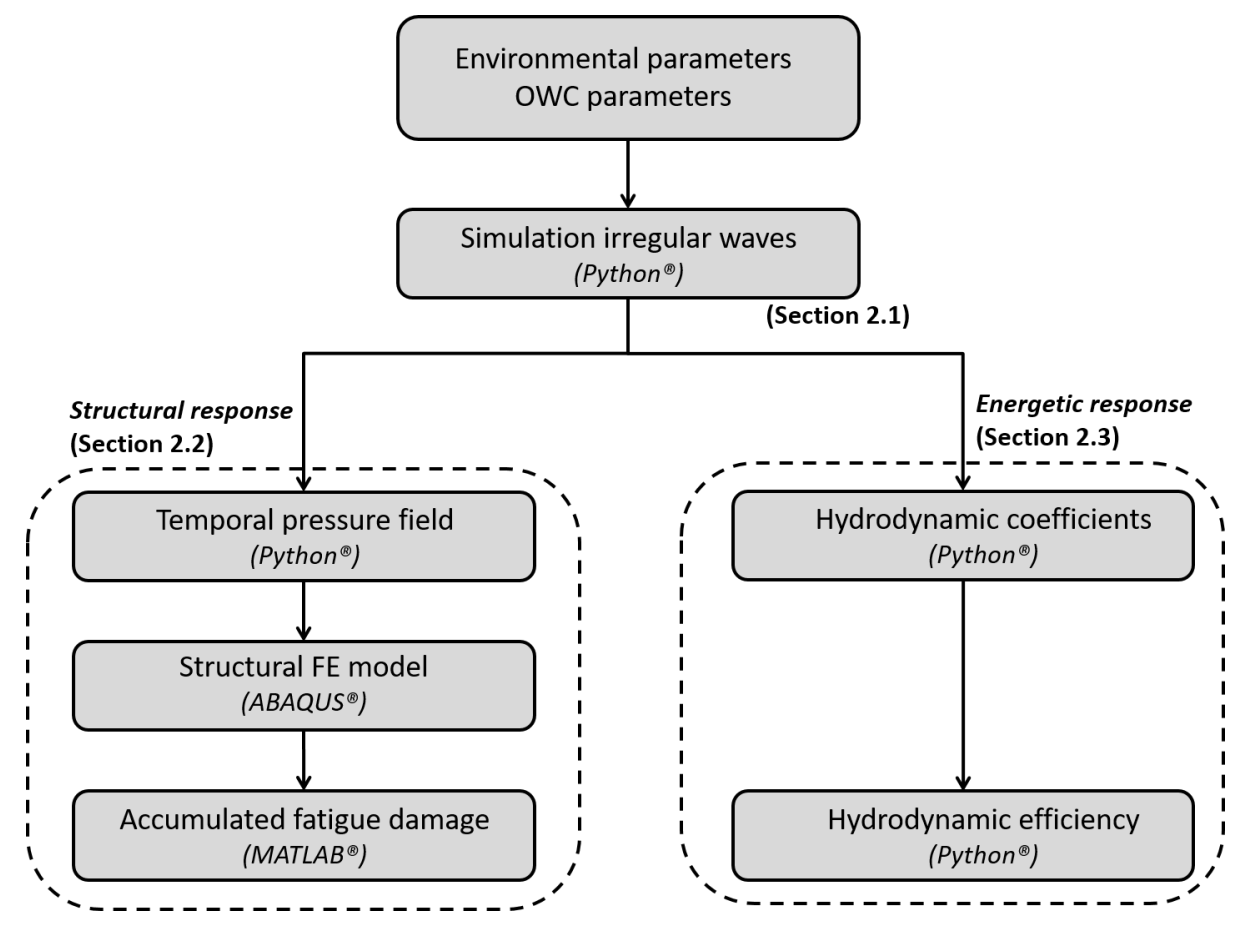

Figure 1: Flowchart of the parameterised computational model. 


\subsection{Description of the problem}

In this paper, the interaction of an incident sea state defined by its significant wave height $H_{s}$, and peak period $T_{p}$, with an idealised bottom-fixed OWC wave energy converter, with radius $a$, draft $d$, emergence $e$, and thin thickness $\xi$, located on the inner continental shelf with constant depth $h$ is considered (Fig. 2). This device has a Wells turbine with rotational speed $N_{s}$ and outer rotor diameter $D_{t}$, and the system can adopt the optimal configuration $\left(d, N_{s}\right)$ for a particular sea state. A detailed description of the real mechanism for such configuration change is out of the scope of this work. The reader is referred to Jalón et al. [13] for further details about this system.

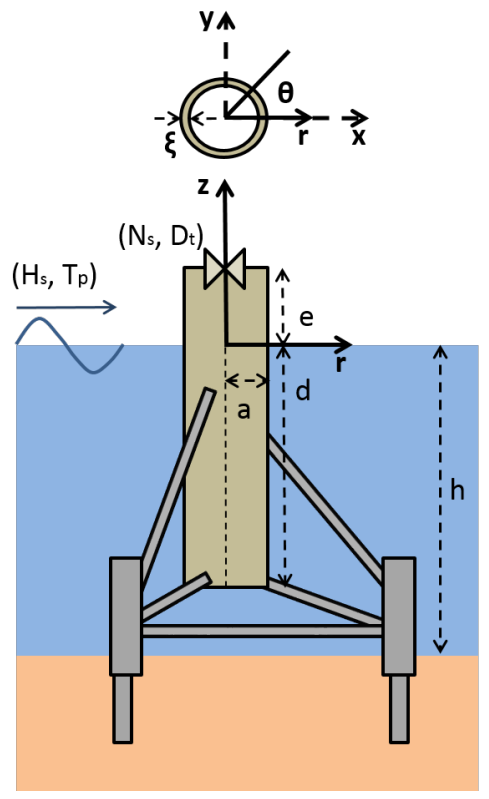

Figure 2: Tentative sketch of the bottom-fixed OWC system.

For the purpose of the fluid-structure interaction, the irregular surface height of ocean waves $\eta$ is assumed to be represented as a sum of a large number $M$ of regular waves with amplitude $A_{j}$, wave number $k_{j}$, wave frequency $f_{j}$, and phase angle $\epsilon_{j}, j=1, \ldots, M$, as [19]:

$$
\eta(x, t)=\sum_{j=1}^{M} \eta_{j}(x, t)=\sum_{j=1}^{M} A_{j} \cos \left(k_{j} x-2 \pi f_{j} t+\epsilon_{j}\right)
$$

where $x$ is the Cartesian coordinate in the direction of the incident wave; $t=\left(0: \Delta t: t_{\max }\right)$, where $t_{\max }$ is the maximum simulated time and $\Delta t$ is the increment of time $\left(\Delta t=1 / 2 \mathrm{f}_{N}\right.$ with $f_{N}$ the Nyquist frequency); and $\epsilon_{j}$ is a random variable uniformly distributed in the range $(0,2 \pi)$. The number of wave components $M$ is determined by the length of the frequency vector $f=\left(0: \Delta f: f_{N}\right)$, where $\Delta f=1 / t_{\max }$. 
The $j$-th wave number $k_{j}$ is calculated from the wave period $T_{j}=1 / f_{j}$, and the depth $h$; whereas the $j$-th wave amplitude $A_{j}$ is calculated from the energy density spectrum curve $S_{\eta}$ and the interval of wave frequency $\Delta f$, as:

$$
A_{j}=\sqrt{2 S_{\eta}\left(f_{j}\right) \Delta f}
$$

In this research, the energy density spectrum curve $S_{\eta}$ is assumed to be given by the JONSWAP wave spectrum [20], thus:

$$
S_{\eta}(f)=\bar{\alpha} g^{2} \frac{1}{f^{5}(2 \pi)^{4}} \exp \left[-1.25\left(\frac{f}{f_{p}}\right)^{-4}\right] \gamma^{\zeta}
$$

with $\gamma=3.3[21] ; \bar{\alpha}=5.058\left[\frac{H_{s}}{T_{p}^{2}}\right]^{2}(1-0.287 \ln \gamma) ; f_{p}=1 / T_{p} ; \zeta=\exp \left[\left(\frac{-\left(f-f_{p}\right)^{2}}{2 \sigma_{j}^{2} f_{p}^{2}}\right)\right] ;$ and $\sigma_{j}=0.07\left(f<f_{p}\right)$ or $\sigma_{j}=0.09\left(f>f_{p}\right)$.

\subsection{Structural response modelling}

\subsubsection{Pressure field in irregular waves}

To calculate the time-dependent pressure field in irregular waves, we assume the irregular sea states (Eq. (1)) as a sequence of regular waves, so that the fluid-structure interaction is formulated as a boundary value problem for regular waves with wave amplitude $A$, wave period $T$, and phase angle $\epsilon$. Assuming incompressible and inviscid fluid, and irrotational motion, the problem is formulated in terms of the velocity potential:

$$
\Phi(r, \theta, z, t)=\Re\left\{\phi_{\text {dif }}(r, \theta, z) e^{-i \omega t}\right\}+\Re\left\{\phi_{\text {rad }}(r, \theta, z) e^{-i \omega t}\right\}
$$

where $r, \theta, z$ are the cylindrical coordinates; $i$ is the imaginary number; $\omega$ is the angular frequency; $\Phi$ is the velocity potential; and $\phi_{d i f}$ and $\phi_{\text {rad }}$ describe the diffraction and radiation effects respectively. This theoretical approach is based on linear wave theory in which the incident wave height is assumed sufficiently small to not consider the nonlinear terms.

The boundary value problem needs to satisfy the Laplace equation, the kinematic boundary condition at the bottom, and the mixed boundary condition on the free surface. Furthermore, the velocity potentials outside and inside the system must satisfy the no flux matching condition around the cylinder, and both velocity potentials must match at their common interface in velocities and potentials. The problem is solved following the methodology of Martins-Rivas and Mei [22], considering the diffraction velocity potentials for a partially immersed circular cylinder described by Garret [23], and the radiation velocity potentials described by Evans and Porter [24]. In order to facilitate the understanding of the paper, a description of the diffraction and radiation velocity potentials is provided in Appendix B.

From this standpoint, the time-dependent pressure field is calculated by assuming the unsteady Bernoulli equation,

$$
\frac{\partial \Phi}{\partial t}+\frac{1}{2}\left[u_{r}^{2}+u_{\theta}^{2}+u_{z}^{2}\right]+\frac{P}{\rho_{w}}+g z=C(t)
$$


where $u_{r}, u_{\theta}, u_{z}$ are the velocity components in the cylindrical coordinates $(r, \theta, z) ; P$ is the time-dependent pressure field; $\rho_{\omega}$ is the water density; $g$ is the gravity acceleration; and $C(t)$ is the Bernoulli's constant.

Considering the Bernoulli's constant as $C(t)=0$, neglecting the nonlinear terms $\left(u_{r}^{2}+u_{\theta}^{2}+u_{z}^{2}=0\right)$, and approximating the pressure value above the mean water level $(0<z \leq \eta)$ by first-order Taylor series at $z=0$ [25], the time-dependent pressure field is obtained as follows:

$$
P(r, \theta, z, t)=\left\{\begin{array}{l}
-\rho_{\omega} g z-\rho_{\omega} \frac{\partial \Phi}{\partial t},-d \leq z \leq 0, \forall r \\
\rho_{\omega} g(\eta-z), 0<z \leq \eta, r>a \\
\rho_{\omega} g(\eta-z)+p, 0<z \leq \eta, r<a
\end{array}\right.
$$

where $a$ is the OWC radius; $\eta$ is the free surface displacement $\left(\eta=-\left.\frac{1}{g} \frac{\partial \Phi}{\partial t}\right|_{z=0}\right)$, and $p$ is the oscillating air pressure inside the system $\left(p(t)=\Re\left\{\widehat{P} e^{-i \omega t}\right\}\right)$ in excess of the atmospheric pressure, which is calculated by applying the model of power take-off for a linear turbine defined in [22].

By specializing Eq. (6) to the region outside the chamber $(r>a)$, the pressure field would be described as:

$$
P_{\text {out }}(r>a, \theta, z, t)=\left\{\begin{array}{l}
-\rho_{\omega} g z-\rho_{\omega}\left(\frac{\partial \Phi_{\text {dif }}(r>a)}{\partial t}+\frac{\partial \Phi_{\text {rad }}(r>a)}{\partial t}\right),-d \leq z \leq 0 \\
\rho_{\omega} g\left(\eta_{\text {dif }}(r>a)+\eta_{\text {rad }}(r>a)-z\right), 0<z \leq \eta
\end{array}\right.
$$

where

$$
\begin{aligned}
& \frac{\partial \Phi_{d i f}(r>a)}{\partial t}=-g \Re\left\{A^{\prime} \sum_{m=0}^{N_{m}} \Gamma_{m}\left[\beta_{m} \frac{Z_{0}(z)}{Z_{0}(0)}+\sum_{l=0}^{N_{l}} B_{m l} \frac{K_{m}\left(k_{l} r\right)}{k_{l} a K_{m}^{\prime}\left(k_{l} a\right)} Z_{l}(z)\right] e^{-i \omega t}\right\} \\
& \frac{\partial \Phi_{r a d}(r>a)}{\partial t}=-\Re\left\{\frac{\widehat{P}}{\rho_{\omega}} \sum_{l=0}^{N_{l}} D_{l} \frac{K_{0}\left(k_{l} r\right)}{k_{l} a K_{0}^{\prime}\left(k_{l} a\right)} Z_{l}(z) e^{-i \omega t}\right\}
\end{aligned}
$$

and

$$
\begin{aligned}
& \eta_{\text {dif }}(r>a)=\Re\left\{A^{\prime} \sum_{m=0}^{N_{m}} \Gamma_{m}\left[\beta_{m}+\sum_{l=0}^{N_{l}} B_{m l} \frac{K_{m}\left(k_{l} r\right)}{k_{l} a K_{m}^{\prime}\left(k_{l} a\right)} Z_{l}(0)\right] e^{-i \omega t}\right\} \\
& \eta_{\text {rad }}(r>a)=\Re\left\{\frac{\widehat{P}}{\rho_{\omega} g} \sum_{l=0}^{N_{l}} D_{l} \frac{K_{0}\left(k_{l} r\right)}{k_{l} a K_{0}^{\prime}\left(k_{l} a\right)} Z_{l}(0) e^{-i \omega t}\right\}
\end{aligned}
$$

Correspondingly, the pressure field in the region inside the chamber $(r<a)$ is obtained as:

$$
P_{i n s}(r<a, \theta, z, t)=\left\{\begin{array}{l}
-\rho_{\omega} g z-\rho_{\omega}\left(\frac{\partial \Phi_{\text {dif }}(r<a)}{\partial t}+\frac{\partial \Phi_{r a d}(r<a)}{\partial t}\right),-d \leq z \leq 0 \\
\rho_{\omega} g\left(\eta_{d i f}(r<a)+\eta_{\text {rad }}(r<a)-z\right)+p, 0<z \leq \eta
\end{array}\right.
$$

where

$$
\begin{aligned}
& \frac{\partial \Phi_{d i f}(r<a)}{\partial t}=-g \Re\left\{A^{\prime} \sum_{m=0}^{N_{m}} \sum_{l=0}^{N_{l}} \Gamma^{m} B_{m l} \frac{I_{m}\left(k_{l} r\right)}{k_{l} a I_{m}^{\prime}\left(k_{l} a\right)} Z_{l}(z) e^{-i \omega t}\right\} \\
& \frac{\partial \Phi_{r a d}(r<a)}{\partial t}=-\Re\left\{\left(\frac{\widehat{P}}{\rho_{\omega}} \sum_{l=0}^{N_{l}} D_{l} \frac{I_{0}\left(k_{l} r\right)}{k_{l} a K_{0}^{\prime}\left(k_{l} a\right)} Z_{l}(z)+\frac{\widehat{P}}{\rho_{\omega}}\right) e^{-i \omega t}\right\}
\end{aligned}
$$


and

$$
\begin{aligned}
& \eta_{\text {dif }}(r<a)=\Re\left\{A^{\prime} \sum_{m=0}^{N_{m}} \sum_{l=0}^{N_{l}} \Gamma_{m} B_{m l} \frac{I_{m}\left(k_{l} r\right)}{k_{l} a I_{m}^{\prime}\left(k_{l} a\right)} Z_{l}(0) e^{-i \omega t}\right\} \\
& \eta_{\text {rad }}(r<a)=\Re\left\{\frac{\widehat{P}}{\rho_{\omega} g} \sum_{l=0}^{N_{l}} D_{l} \frac{I_{0}\left(k_{l} r\right)}{k_{l} a I_{0}^{\prime}\left(k_{l} a\right)} Z_{l}(0) e^{-i \omega t}\right\}
\end{aligned}
$$

The reader is referred to the Nomenclature table and the Appendix B for further insight about the undefined terms appearing in Eqs. (8a) to (10d).

\subsubsection{Accumulated fatigue damage}

A structural finite-element (FE) model, which idealises the structural response of the interaction between the incident sea state and the bottom-fixed OWC, is developed using ABAQUS ${ }^{\circledR}$. The modeled bottom-fixed OWC is represented by a shell-type cylindrical structure with radius $a$, draft $d$, thickness $\xi$, and emergence $e$. As a modelling assumption, the support structure is not considered in the fluid-structure interaction, so four fixed points at the bottom of the system of the cylinder are conservatively adopted as boundary conditions to represent the support structure. Further geometrical and mechanical details are provided in Section 3.3.2. Apart from the gravity and the sub-pressure, the time-dependent pressure field developed in Section 2.2.1 (Eq. (6)) is applied as input load. The contribution of the oscillating air pressure inside the air chamber $(\eta<z \leq e)$ to the structural response of the system is neglected as it was revealed as insignificant after some pilot tests.

The FE model is run for $t=\left(0: \Delta t: t_{\max }\right)$, where $t_{\max }$ is the maximum simulated time (Eq. (1)), and time series of the stresses measured at a set of representative points are obtained as model outputs. Then, the rainflow counting algorithm is applied to these time series to determine the number of cycles $n_{i}$ corresponding to the $i$-th stress range $\Delta \sigma_{i}, i=1, \ldots, s$. Finally, by applying the Palmgren-Miner's rule, the accumulated fatigue damage $D$ is obtained as [26]:

$$
D=\frac{1}{\bar{a}} \sum_{i=1}^{s} n_{i} \Delta \sigma_{i}^{m}
$$

where $m$ and $\bar{a}$ are the empirical fatigue properties obtained from the $\mathrm{S}-\mathrm{N}$ curve of the material, which is assumed as known in this research.

\subsection{Energetic response modelling}

The most important parameter of the performance of an OWC is the hydrodynamic efficiency, which is represented by the dimensionless number $k L$ as:

$$
k L=k \frac{\bar{P}_{a v a i}}{\bar{P}_{\omega}}
$$


where $k$ is the wave number, and $L$ is the capture length, obtained as the ratio between the time-averaged power extracted from the wave $\bar{P}_{\text {avai }}$ and the incident energy flux per unit wave crest length $\bar{P}_{\omega}$. $\bar{P}_{\text {avai }}$ represents the available pneumatic power used by the linear turbine to generate mechanical power, whereas $\bar{P}_{\omega}$ represents the available wave power at a sea state. Both powers are calculated in irregular waves as [6, 13]:

$$
\begin{gathered}
\bar{P}_{a v a i}=\frac{K D_{t} g^{2} \rho_{\omega}^{2}}{N_{s} \rho_{a}} \int_{0}^{\infty} S_{\eta}(f) F(f) d f \\
\bar{P}_{\omega}=\rho_{\omega} g \int_{0}^{\infty} S_{\eta}(f) C_{g}(f) d f
\end{gathered}
$$

In Eq. $(14), S_{\eta}(f)$ is the energy density spectrum; and $C_{g}(f)$ is the group velocity of the sea state at a specific frequency $f$; whereas in Eq. (13) $K$ is an empirical factor that depends on the design, number, and configuration of the turbines; $D_{t}$ is the outer diameter of the turbine rotor; $N_{s}$ is the rotational speed; and $\rho_{a}$ is the static air density. The term $F(f)$ in Eq. (13) is defined by:

$$
F(f)=\frac{|\widetilde{\Gamma}(f)|^{2}}{(\chi(f)+\widetilde{B}(f))^{2}+(\beta(f)+\widetilde{C}(f))^{2}}
$$

where $\widetilde{\Gamma}(f), \widetilde{B}(f), \widetilde{C}(f)$ are the dimensionless hydrodynamic coefficients; and $\chi(f)$ and $\beta(f)$ represent the turbine and the air compressibility in the chamber at a specific frequency, respectively. These terms are described in [22], and they are obtained by the analytical model developed in Section 2.2.1.

Consequently, by substituting Eqs. (13) and (14) in Eq. (12), the hydrodynamic efficiency in irregular waves can be calculated as:

$$
k L=\frac{K D_{t} g \rho_{\omega}}{N_{s} \rho_{a}} \frac{\int_{0}^{\infty} k(f) S_{\eta}(f) F(f) d f}{\int_{0}^{\infty} S_{\eta}(f) C_{g}(f) d f}
$$

Thus, for any wave climate data $\left(H_{s}, T_{p}, h\right)$ and any OWC configuration $\left(d, a, e, D_{t}, N_{s}\right)$, Eq. (16) can be used to obtain the hydrodynamic efficiency of the system in irregular waves through a spectral approach.

\section{Illustrative case study}

The proposed methodology is exemplified for a particular bottom-fixed OWC device located in a constant depth $h=10 \mathrm{~m}$, with radius $a=3.5 \mathrm{~m}$, emergence $e=5 \mathrm{~m}$, thickness $\xi=0.02 \mathrm{~m}$, turbine diameter $D_{t}=$ $1 \mathrm{~m}$, and empirical factor $K=0.45$. The analyzed sea states $\left(\mathrm{S}_{1}, \mathrm{~S}_{2}, \mathrm{~S}_{3}\right)$ and configurations (Cases A to F) showed in Table 1 are adopted from Jalón et al. [13], and represent sea state and annual power-optimal configurations in a specific location of the Gulf of Cadiz (Spain). In particular, Cases A, B, C represent the sea state power-optimal configurations associated to the sea states $\mathrm{S}_{1}, \mathrm{~S}_{2}, \mathrm{~S}_{3}$, respectively, whereas Cases D, E, F represent the annual power-optimal configuration for the states $\mathrm{S}_{1}, \mathrm{~S}_{2}, \mathrm{~S}_{3}$. 


\begin{tabular}{lcc|ccc|ccc}
\hline & \multicolumn{3}{c}{ Sea state } & \multicolumn{4}{c}{ Sea state optimal configuration } & \multicolumn{2}{c}{ Annual optimal configuration } \\
\hline Case & $\mathrm{H}_{s}(m)$ & $\mathrm{T}_{p}(s)$ & Case & $\mathrm{d}(\mathrm{m})$ & $\mathrm{N}_{s}($ rpm $)$ & Case & $\mathrm{d}(\mathrm{m})$ & $\mathrm{N}_{s}(\mathrm{rpm})$ \\
\hline $\mathrm{S}_{1}$ & 1.17 & 4.22 & $\mathrm{~A}$ & 2.0 & 184 & $\mathrm{D}$ & 8.0 & 294.14 \\
$\mathrm{~S}_{2}$ & 1.64 & 5.43 & $\mathrm{~B}$ & 5.0 & 154 & $\mathrm{E}$ & 8.0 & 294.14 \\
$\mathrm{~S}_{3}$ & 2.10 & 7.86 & $\mathrm{C}$ & 8.0 & 212 & $\mathrm{~F}$ & 8.0 & 294.14 \\
\hline
\end{tabular}

Table 1: Sea states $\left(\mathrm{S}_{1}, \mathrm{~S}_{2}, \mathrm{~S}_{3}\right)$ and power-optimal configurations (Cases A to F) of the bottom-fixed OWC device [13].

\subsection{Simulation of irregular sea waves}

For the sea states $\left(\mathrm{S}_{1}, \mathrm{~S}_{2}, \mathrm{~S}_{3}\right)$ defined in Table 1, simulated incident wave profiles (Eq. (1)) are obtained for $t_{\max }=3600 \mathrm{~s}$ and increment of time $\Delta t=0.33 \mathrm{~s}$. In order to apply the analytical formulation developed in Section 2.2.1, the assumption that an irregular sea state can be considered as a sequence of regular sea waves is adopted. To illustrate the validity of such an assumption, the energy density spectra for both the simulated incident wave profile as a sequence of regular waves $\left(\mathrm{S}_{i, r e g}, i=1,2,3\right)$ and that considering irregular sea states $\left(\mathrm{S}_{i, i r r}, i=1,2,3\right)$ are represented in Fig. 3a. Moreover, the cumulative distribution function $(\mathrm{CDF})$ of the wave amplitudes for the irregular sea states considered as a sequence of regular sea waves is represented in Fig. 3b. In view of the results, the adopted assumption can be considered as valid since the spectra are quite similar and the wave amplitudes follow a Rayleigh distribution, as they are supposed to follow for a narrow-banded sea spectrum [27].

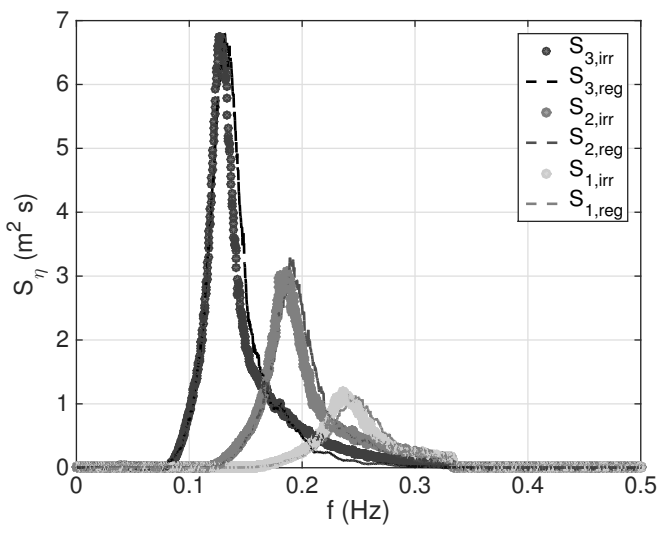

(a)

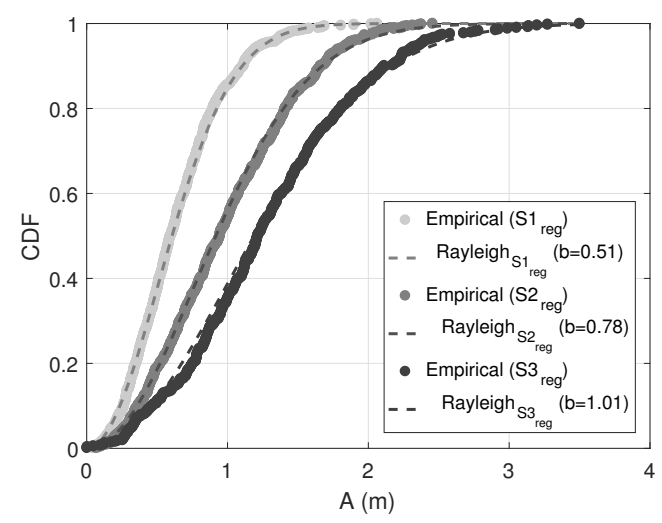

(b)

Figure 3: (a) Energy density spectra. (b) Empirical (dots) and theoretical (dashed line) cumulative distribution functions. $\mathrm{S}_{1}: H_{s}=1.17 \mathrm{~m}$ and $T_{p}=4.22 \mathrm{~s}, \mathrm{~S}_{2}: H_{s}=1.67 \mathrm{~m}$ and $T_{p}=5.43 \mathrm{~s}, \mathrm{~S}_{3}: H_{s}=2.1 \mathrm{~m}$ and $T_{p}=7.86 \mathrm{~s}$. 


\subsection{Energetic response modelling}

In accordance with the theory in Section 2.3, Table 2 shows the results for the total available pneumatic power $\bar{P}_{\text {avai }}$ (Eq. (13)), the total wave energy $\bar{P}_{w}$ (Eq. (14)), and the hydrodynamic efficiency $k L$ (Eq. (16)) from each of the configurations from Cases A to F (Table 1).

Table 2: Value of the wave energy, pneumatic power and hydrodynamic efficiency.

\begin{tabular}{cccc}
\hline Case & $\bar{P}_{w}(k W / m)$ & $\bar{P}_{\text {avai }}(k W)$ & $\mathrm{kL}$ \\
\hline $\mathrm{A}\left(\mathrm{S}_{1}\right)$ & 2.45 & 6.14 & $\mathbf{0 . 6 3 0 4}$ \\
$\mathrm{B}\left(\mathrm{S}_{2}\right)$ & 7.19 & 21.15 & 0.4509 \\
$\mathrm{C}\left(\mathrm{S}_{3}\right)$ & 17.54 & 39.98 & 0.2342 \\
$\mathrm{D}\left(\mathrm{S}_{1}\right)$ & 2.45 & 0.6 & $\mathbf{0 . 0 4 1 1}$ \\
$\mathrm{E}\left(\mathrm{S}_{2}\right)$ & 7.19 & 10.89 & 0.2034 \\
$\mathrm{~F}\left(\mathrm{~S}_{3}\right)$ & 17.54 & 40.10 & 0.2312 \\
\hline
\end{tabular}

As evident from the results in Table 2, the less energetic sea state $\left(\mathrm{S}_{1}\right)$ with an annual optimal configuration of the system (Case D) yields the lowest pneumatic power and the lowest efficiency. To the contrary, the more energetic sea state $\left(\mathrm{S}_{3}\right)$ with an annual optimal configuration of the system (Case $\mathrm{F}$ ) yields the highest pneumatic power although does not provide the largest hydrodynamic efficiency. The most efficient system corresponds with the less energetic sea state $\left(S_{1}\right)$ with a sea state optimal configuration (Case A) reaching $63.04 \%$ of capture.

\subsection{Structural response modelling}

\subsubsection{Pressure field by irregular waves}

As shown in Section 2.2.1, the time-dependent pressure field (Eq. (6)) depends on the velocity potential $\Phi$, and the expressions of the velocity potential (Eqs. (B.1) and (B.2)) depend on the expansion coefficients $N_{m}$, and $N_{l}$. In this sense, a methodology analogous to the proposed in Jalón et al. [28] is adopted here to obtain the minimum required values for $N_{m}$ and $N_{l}$. Specifically, the radial velocity $U$ at $r=$ a, $\theta=\pi, z=$ $0 \mathrm{~m}$, and $t=0 \mathrm{~s}$ for the annual optimal configuration with an incident wave with amplitude $A=0.1 e^{i \pi} \mathrm{m}$, and period $T=3 \mathrm{~s}$, is calculated for different values of $N_{m}$, and $N_{l}$ in Fig. 4 . It is observed that the solution converges through non velocity $(U=0)$ at $N_{m}=8$, and $N_{l}=250$ with an error of $\mathrm{O}\left(10^{-5}\right)$, therefore, the results presented henceforth are calculated with these values. 


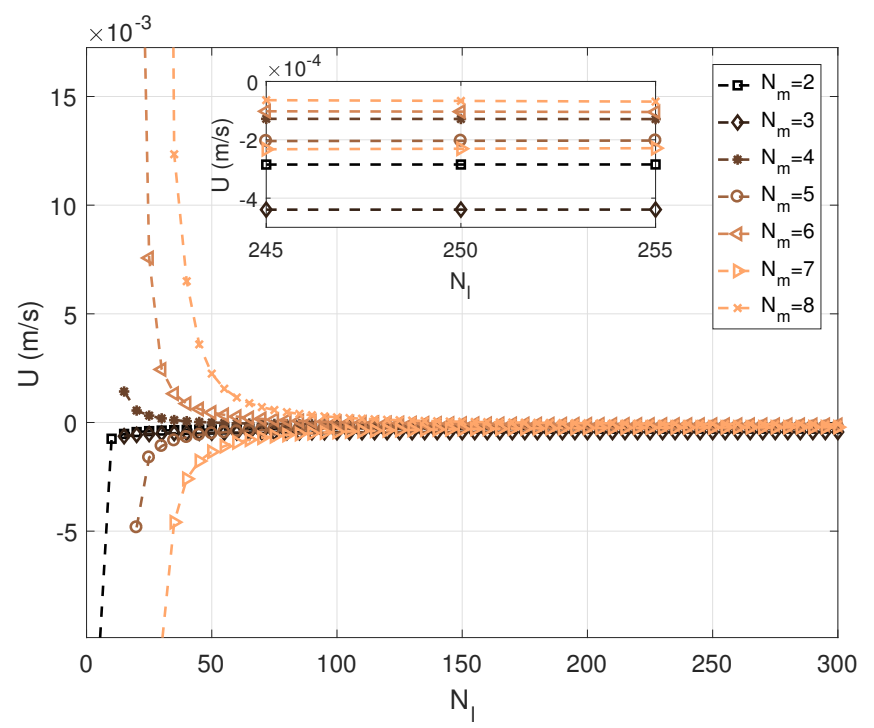

Figure 4: Radial velocity at $r=\mathrm{a}, \theta=\pi, z=0 \mathrm{~m}$, and $t=0 \mathrm{~s}$, for different values of $N_{l}$ and $N_{m}$. Annual optimal OWC configuration.

Then, Eqs. (7) and (9) are applied to obtain the temporal pressure field for Cases A and D, since these cases have been shown to yield the largest and lowest capture, respectively (refer to Table 2). Therefore, for the sake of simplicity, the subsequent results are limited to these two representative case studies. According to Eq. (6), the time-dependent pressure field for $z=0 \mathrm{~m}$ is given by the free surface displacement $\eta$, and the oscillating air pressure $p$. Therefore, for illustrative purposes, the free surface elevation outside $\left(\eta_{\text {out }}\right)$ and inside $\left(\eta_{\text {ins }}\right)$ of the system $(r=a)$ and the oscillating air pressure $(p)$ along time at $\theta=180^{\circ}$, are represented in Figs. 5 and 6 respectively. As can be observed, $\eta_{\text {out }}$ is equal for both Cases A (largest capture) and D (lowest capture); however, $\eta_{i n s}$ and $p$ are significantly larger for the sea state optimal configuration (Case A). Therefore, the inner pressure field $\left(P_{i n s}\right)$ will be higher in Case A than in Case D, as shown in Fig. 7. Correspondingly, the total pressure field $\left(P_{\text {out }}-P_{\text {ins }}\right)$ will be lower in Case A than in Case $\mathrm{D}$, and consequently Case D will lead to higher stresses, as will be shown further below. 


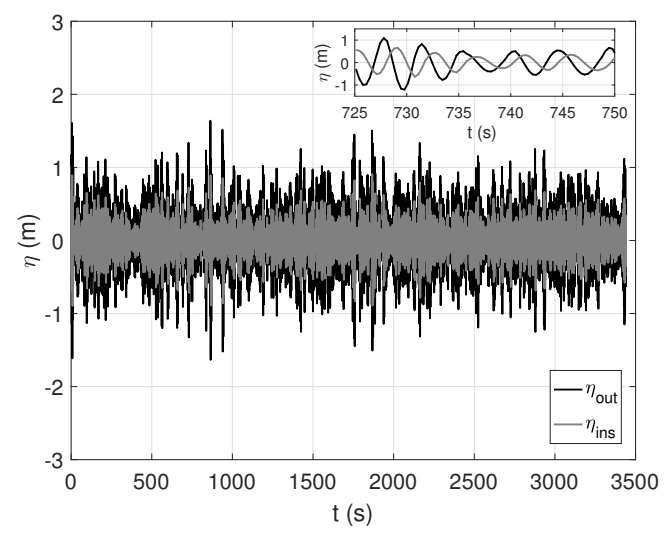

(a) Case $\mathrm{A}$

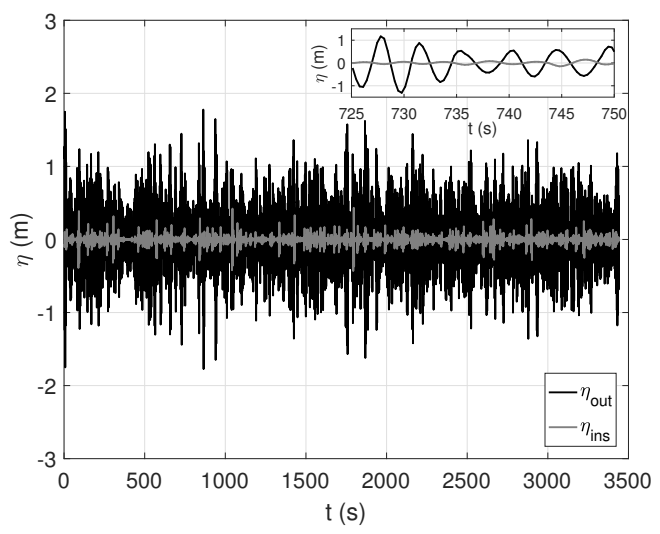

(b) Case D

Figure 5: Free surface displacement outside and inside the system at $r=a, \theta=180^{\circ}$. (a) Case A: Sea state optimal configuration, (b) Case D: Annual optimal configuration. $\mathrm{S}_{1}: H_{s}=1.17 \mathrm{~m}$ and $T_{p}=4.22 \mathrm{~s}$.

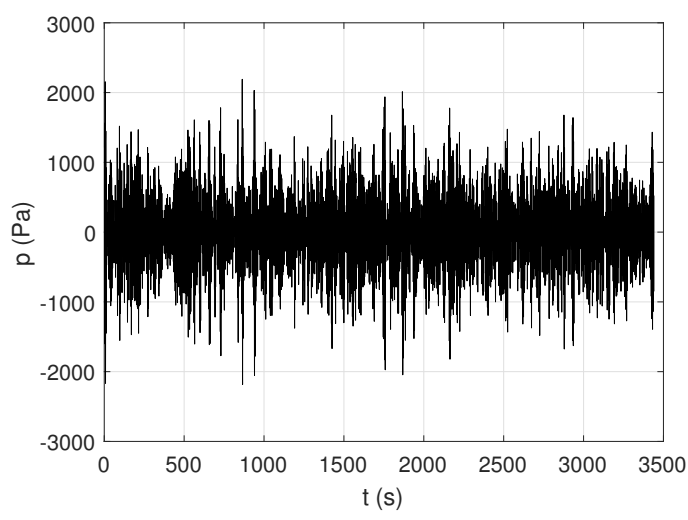

(a) Case A

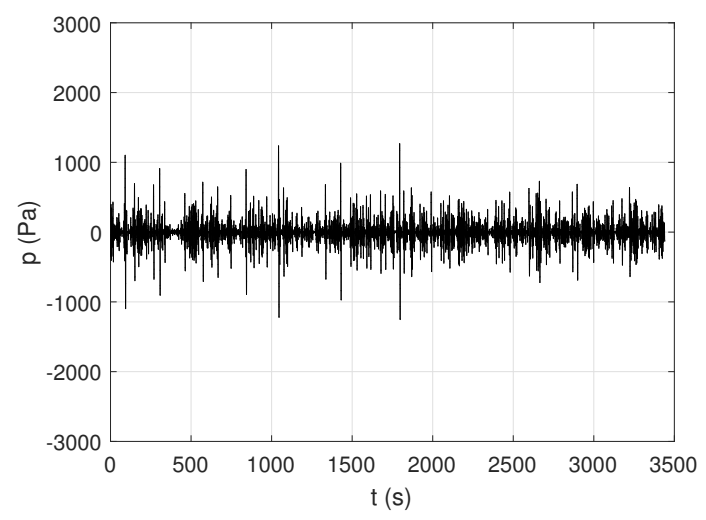

(b) Case D

Figure 6: Oscillating air pressure at $r=a, \theta=180^{\circ}$.(a) Case A: Sea state optimal configuration, (b) Case D: Annual optimal configuration. $\mathrm{S}_{1}: H_{s}=1.17 \mathrm{~m}$ and $T_{p}=4.22 \mathrm{~s}$. 


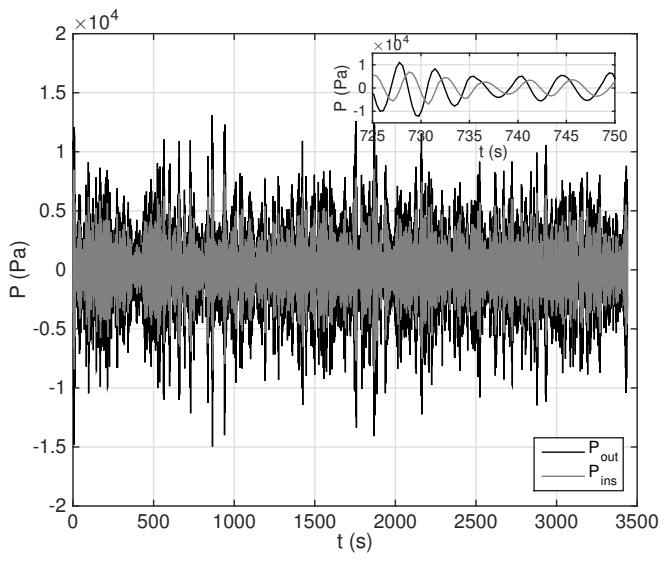

(a) Case A

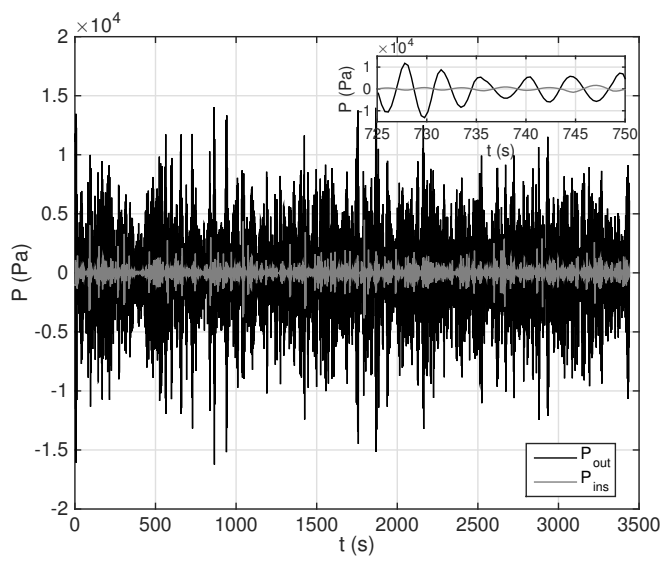

(b) Case D

Figure 7: Pressure field inside and outside the system at $r=a, \theta=180^{\circ}, z=0 \mathrm{~m}$. (a) Case A: Sea state optimal configuration, (b) Case D: Annual optimal configuration. $\mathrm{S}_{1}: H_{s}=1.17 \mathrm{~m}$ and $T_{p}=4.22 \mathrm{~s}$.

The results in Fig. 7 provide information about the time-dependent pressure field at a specific point. However, the total pressure field around the OWC is also investigated here since the stresses at a specific point depend not only on the total pressure at that point, but also on the whole pressure field. To this end, Eqs. (7) and (9) are solved for a particular time $(t=728.4 \mathrm{~s})$ at $r=a$, and the total pressure field $\left(P_{\text {total }}=P_{\text {out }}-P_{\text {ins }}\right)$ is obtained and represented in Fig. 8. In view of the results, Case D leads to higher total pressures than Case A for $\theta=[90,270]$; consequently, Case D will yield higher stresses and therefore higher values of stress ranges. It is observed also that the total pressure field presents a planar symmetry respect to $\theta=180^{\circ}$, (because the diffraction effect depends on $\cos (m \theta), m=0, \ldots, N_{m}$ ); thus, the structural analysis can be simplified by using a symmetric model. 


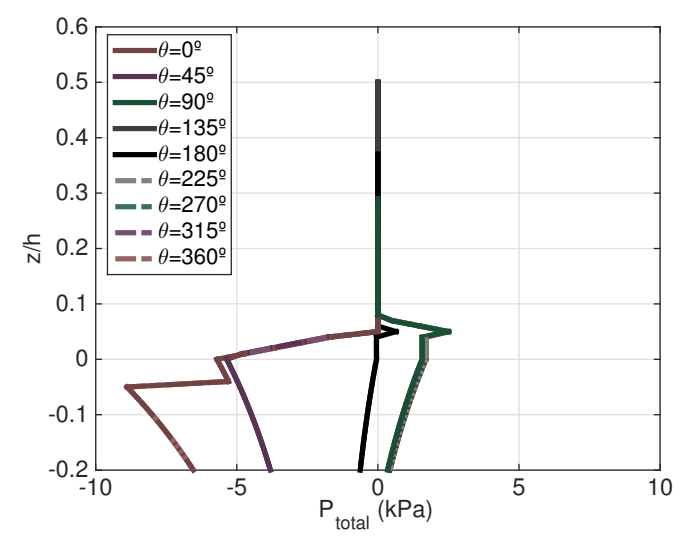

(a) Case A

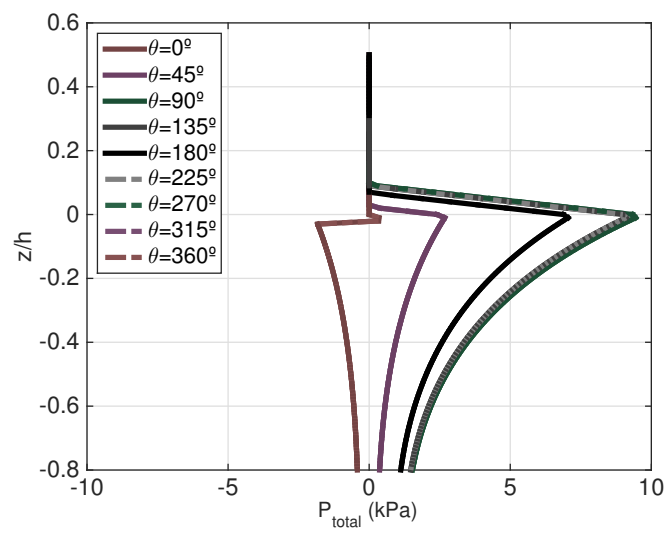

(b) Case D

Figure 8: Total pressure field at $t=728.4 \mathrm{~s}, r=a$. (a) Case A: Sea state optimal configuration, (b) Case D: Annual optimal configuration. $\mathrm{S}_{1}: H_{s}=1.17 \mathrm{~m}$ and $T_{p}=4.22 \mathrm{~s}$.

\subsubsection{Accumulated fatigue damage}

Regarding the FE model, a shell-type cylindrical structure with radius $a=3.5 \mathrm{~m}$, thickness $\xi=0.02 \mathrm{~m}$, emergence $e=5 \mathrm{~m}$, and different drafts $d$ is modeled in ABAQUS ${ }^{\circledR}$, using a linear elastic material (steel) with Young's modulus $E=210 \mathrm{GPa}$, and Poisson ratio $\nu=0.3$. The adopted element is the S4R [29] and the mesh size is $0.25 \mathrm{~m}$, appropriately selected after a mesh convergence study (not shown here for the sake of conciseness), such that the solution given by ABAQUS ${ }^{\circledR}$ becomes independent of the mesh size. Four fixed points located at $\theta=\left\{45^{\circ}, 135^{\circ}, 225^{\circ}, 315^{\circ}\right\}$ at the base of the cylinder, are adopted as boundary conditions representing the support structure of the OWC. Fig. 9 illustrates the structural FE model of the annual optimal configuration with the pressure field at $\mathrm{t}=728.4 \mathrm{~s}$. 


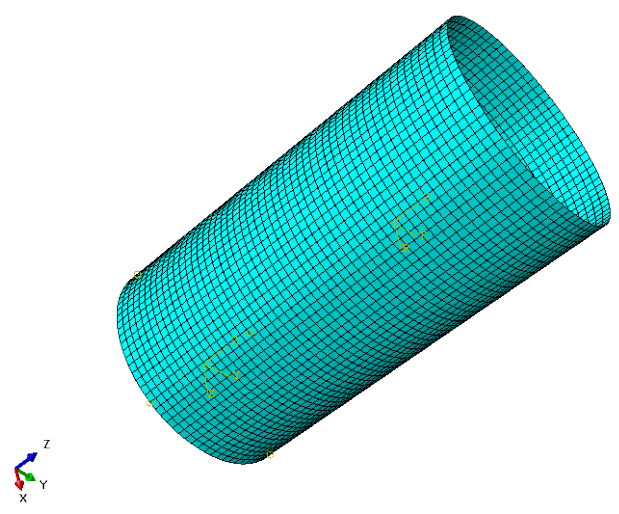

(a)

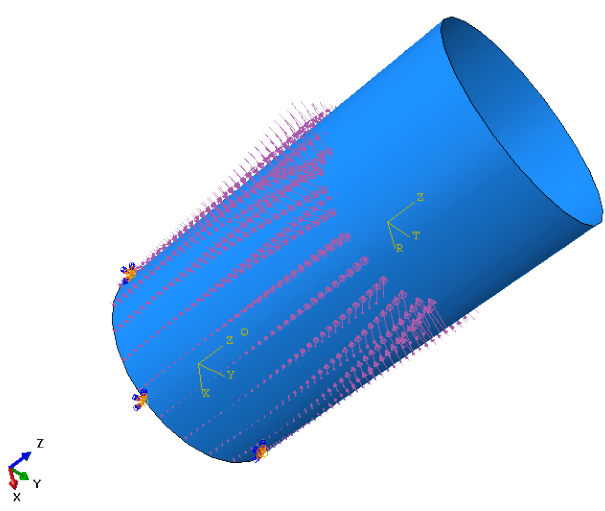

(b)

Figure 9: Structural FE model of the annual optimal configuration. (a) Mesh details. (b) Pressure field at t $=728.4$ s. S1: $H_{s}=1.17$ $\mathrm{m}, T_{p}=4.22 \mathrm{~s}$.

Based on the planar symmetry derived from the total pressure field (Fig. 8), the maximum principal stresses $\sigma(t)$ are measured for $\theta=\left\{0^{\circ}, 90^{\circ}, 180^{\circ}\right\}$ at different depths $z=(0:-1:-d+1)$. Note that the maximum principal stress component is used for fatigue calculation since this is the component of stress recommended to use with the fatigue design curves. Then, the stress ranges $\Delta \sigma_{i}$ and the corresponding number of cycles $n_{i}$ are determined from $\sigma(t)$ by applying the rainflow cycle counting algorithm with WAFO (Wave Analysis for Fatigue and Oceanography), a MATLAB ${ }^{\circledR}$ toolbox for statistical analysis and simulation of random loads [30]. In this sense, the rainflow matrix (RFM), which shows the number of cycles $n_{i}$ corresponding to the applied stress range $\Delta \sigma_{i}$, is obtained using a discretization level which follows the Sturges approximation [31]. Figures 10a, 10b, and 11, show the maximum principal stresses, the CDF of the stress ranges, and the RFM for Cases A and D, respectively, for $\theta=180^{\circ}$, and $z=0 \mathrm{~m}$. 


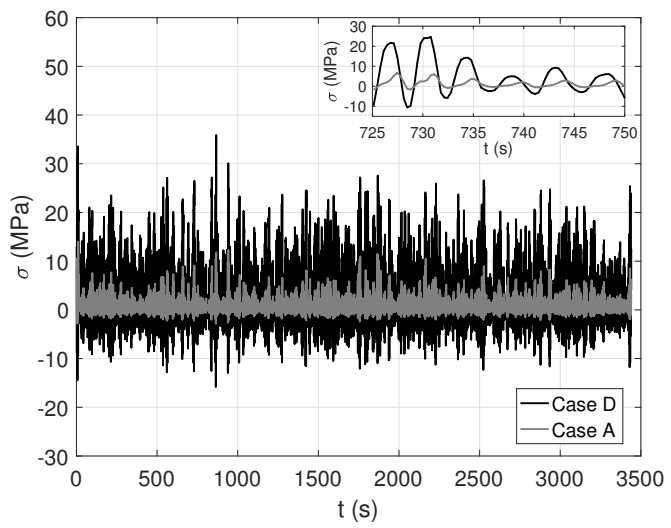

(a)

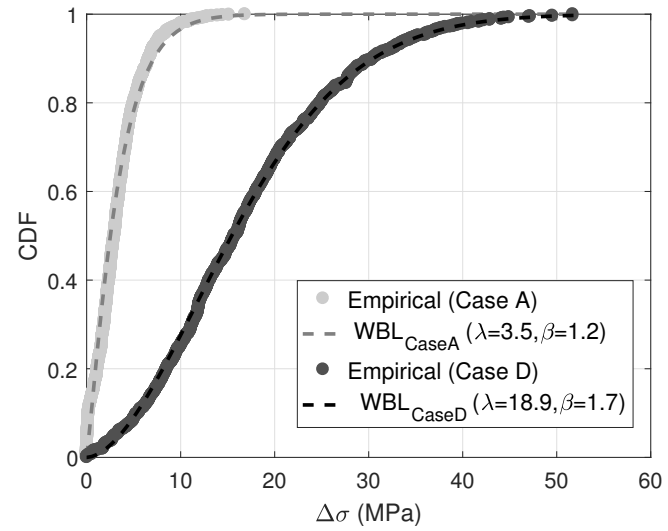

(b)

Figure 10: (a) Time history of the maximum principal stresses at $\theta=180^{\circ}$ and $z=0 \mathrm{~m}$. (b) Theoretical (dashed line) and empirical (dots) cumulative distribution functions for the stress ranges. Case A-Case D. $\mathrm{S}_{1}: H_{s}=1.17 \mathrm{~m}$ and $T_{p}=4.22 \mathrm{~s}$.

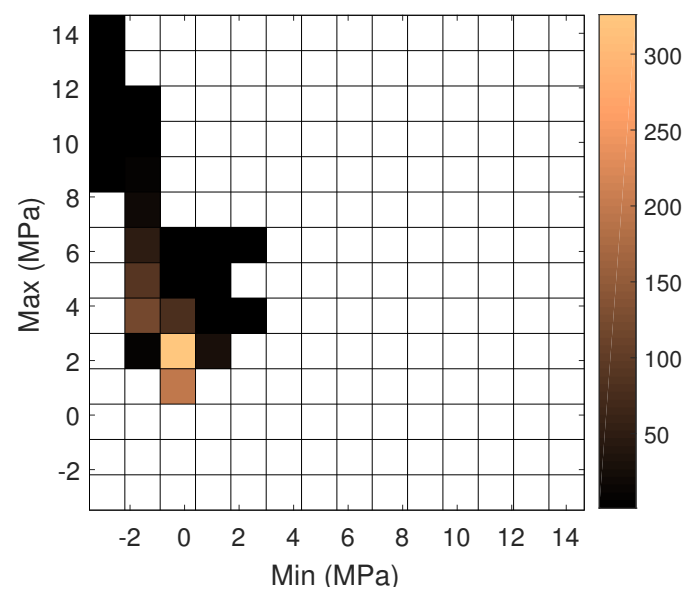

(a) Case A

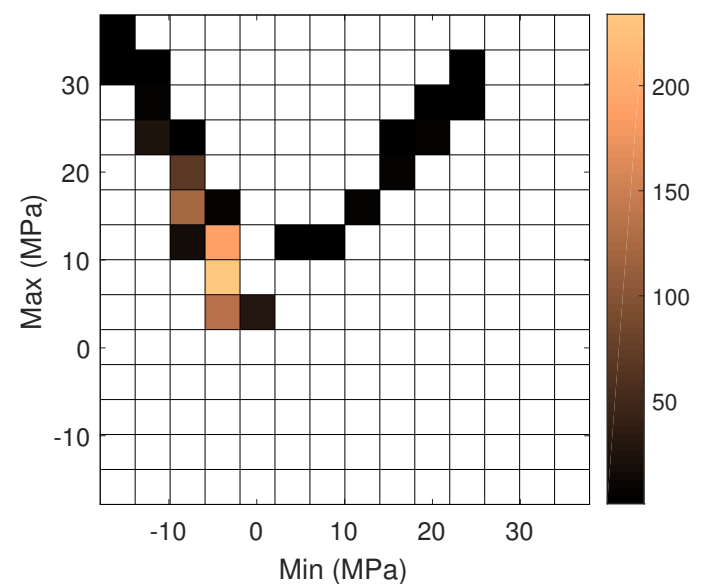

(b) Case D

Figure 11: Rainflow matrix at $\theta=180^{\circ}$ and $z=0 \mathrm{~m}$. (a) Case A. (b) Case D. $\mathrm{S}_{1}: H_{s}=1.17 \mathrm{~m}$ and $T_{p}=4.22 \mathrm{~s}$.

As is evident from Figs. 10a, 10b and 11b, the maximum stresses and the stress ranges arise in Case D (annual optimal configuration). This is due to the higher difference between the pressure field inside and outside the system at $\theta=180^{\circ}$, as it is observed in Fig 7. Consequently Case D will lead to higher values of accumulated fatigue damage although it is subjected to less load cycles than Case A (Fig. 11). In regard to the stress ranges (Fig. 10b), their CDFs follow a Weibull distribution with scale $(\lambda)$ and shape $(\beta)$ parameters. In addition, note that the full stress ranges are calculated considering the tension and compression as is normal practice for welded steel structures. 
Finally, the accumulated fatigue damage $D$ (Eq. (11)) is calculated based on the RFM and the fatigue properties of the material. To this end, the S-N Curve D for steel in seawater with cathodic protection [32], with fatigue properties $\log \bar{a}=11.764, m=3$ if $N \leq 10^{6}$ cycles, and $\log \bar{a}=15.606, m=5$ otherwise, is adopted as a conservative design assumption. Figure 12 shows the accumulated fatigue damage as a function of the cylindrical coordinates $z / d$ and $\theta$. 


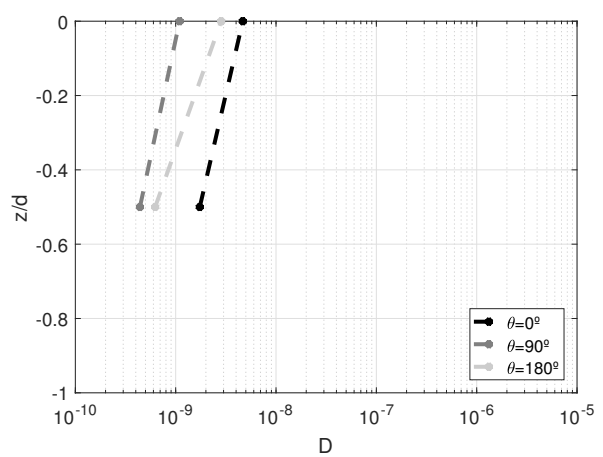

(a) Case A

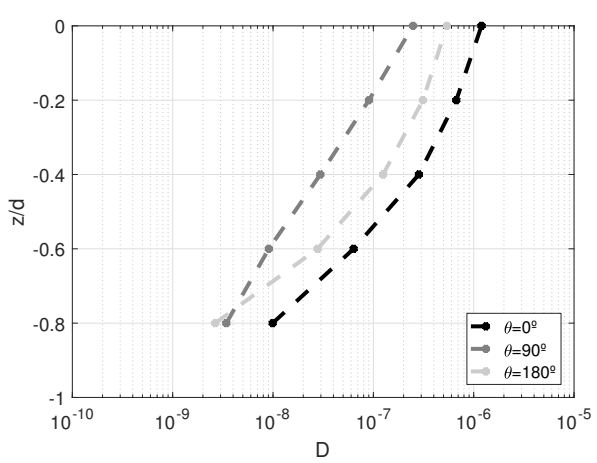

(c) Case B

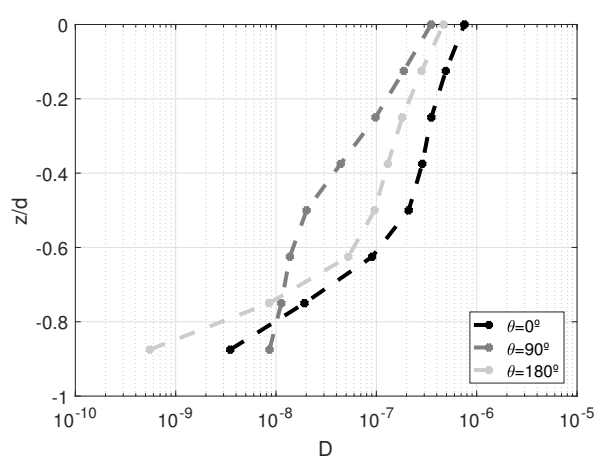

(e) Case C

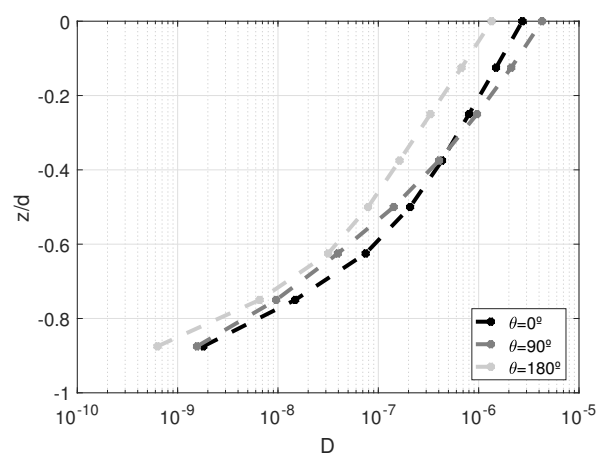

(b) Case D

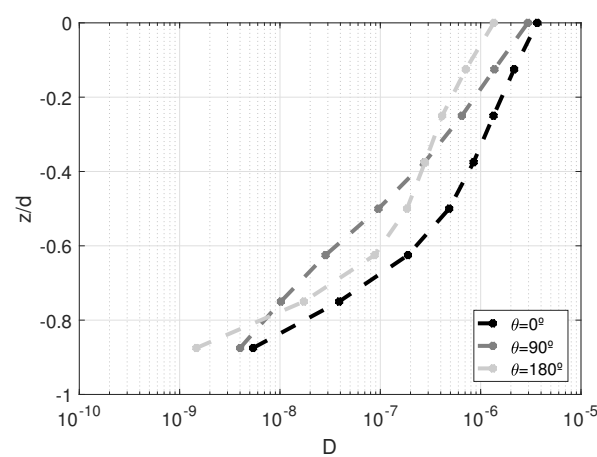

(d) Case E

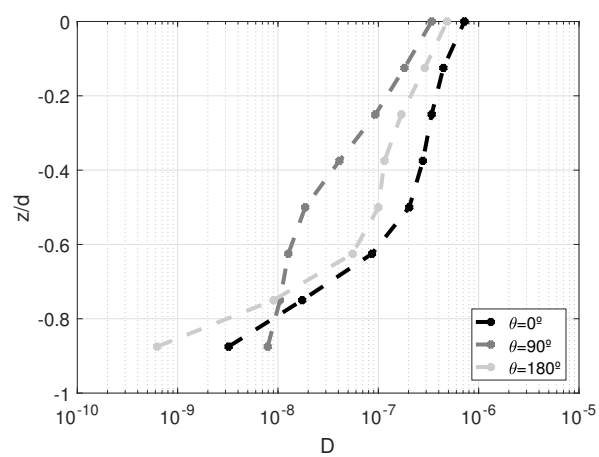

(f) Case F

Figure 12: Accumulated fatigue damage. (a) Case A $\left(\mathrm{S}_{1}\right)$, (b) Case D $\left(\mathrm{S}_{1}\right)$, (c) Case B $\left(\mathrm{S}_{2}\right)$, (d) Case E $\left(\mathrm{S}_{2}\right)$, (e) Case C $\left(\mathrm{S}_{3}\right)$, (f) Case F $\left(\mathrm{S}_{3}\right)$.

It can be seen that the accumulated fatigue damage reaches the highest values at low values of depth $(z / d \approx 0)$, irrespectively of $\theta$. This is due to the fact that the total pressure field yields the highest values at $z / d \approx 0$ (see Fig. 8). Regarding to the variability of damage for different orientations $\theta$ around the cylinder, results show that the maximum accumulated fatigue damage at $z / d \approx 0$ takes place for $\theta=0^{\circ}$, except Case 
D (Fig. 12b) where the maximum damage happens to take place at $\theta=90^{\circ}$.

Furthermore, it is observed that Cases C (Fig. 12e) and F (Fig. 12f) yield very similar accumulated fatigue damage profiles. These cases are simulated for the same sea state and draft, being the only difference the rotational speed. Therefore, it can preliminary concluded that the rotational speed has not effect on the accumulated fatigue damage for these analyzed Cases C and F.

In addition, it is noted from Figs. 12d and $12 \mathrm{f}$ that the sea state with a medium energetic content $\left(S_{2}\right)$ yields more damage than the sea state with the highest energetic content $\left(S_{3}\right)$ for the same annual optimal configuration (Cases $\mathrm{E}$ and $\mathrm{F})$. As can be observed from the temporal pressure field inside $\left(P_{\text {ins }}\right)$ and outside $\left(P_{\text {out }}\right)$ the system in Fig. 13, $P_{\text {ins }} \approx P_{\text {out }}$ in Case E, whereas $P_{\text {ins }}>\mathrm{P}_{\text {out }}$ in Case F. However, a phase lag of $\varphi \approx \pi$ between $P_{\text {ins }}$ and $P_{\text {out }}$ is observed in Case E, which amplifies the total pressure field $P_{\text {out }}-P_{\text {ins }}$ thus leading to a higher accumulated fatigue damage.

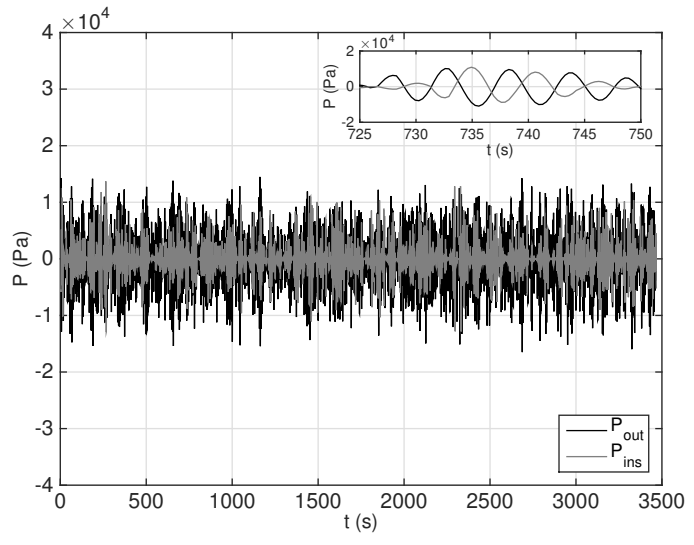

(a) Case E

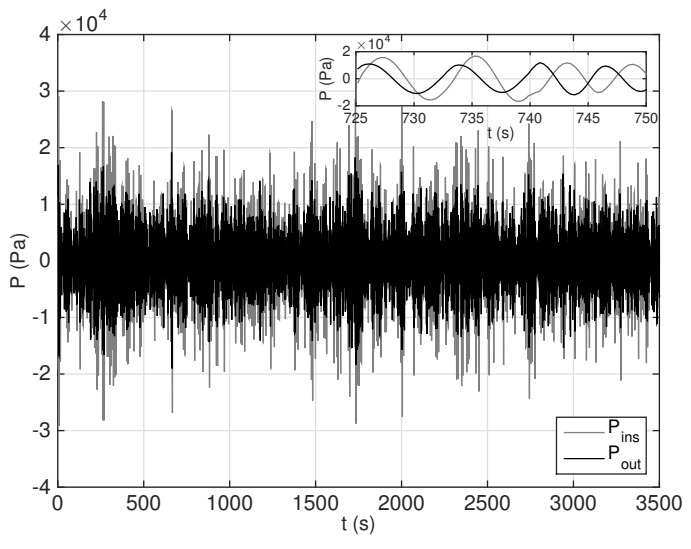

(b) Case F

Figure 13: Pressure field inside and outside the system at $r=a, \theta=180^{\circ}$, and $z / d=0$. (a) Case E, (b) Case F.

\subsection{Hydrodynamic efficiency versus accumulated fatigue damage}

In this section, the influence of the system configuration $\left(d, N_{s}\right)$ on the system performance considering both the fatigue damage and the hydrodynamic efficiency is investigated. To this end, the following two configurations are analyzed for the three considered sea states (namely, $\mathrm{S}_{1}, \mathrm{~S}_{2}$ and $\mathrm{S}_{3}$ ):

1) OWC device capable of automatically adopting the optimal configuration for a particular sea state.

2) OWC device with fixed optimal-annual configuration.

For these two configurations, the hydrodynamic efficiency $k L$ and the fatigue damage $D$ are obtained in a cumulative way, i.e. $k L_{i}=\sum_{j=1}^{i} 0.33 k L\left(S_{j}\right)$ and $D_{i}=\sum_{j=1}^{i} D\left(S_{j}\right), i=1,2,3$. In particular, the dimensionless values $\widetilde{k L}_{i}(\mathrm{Eq} 17 \mathrm{a}), \widetilde{D}_{i}(\mathrm{Eq} 17 \mathrm{~b})$ are calculated to compare between the referred configurations: 


$$
\begin{aligned}
\widetilde{k L_{i}} & =\frac{k L_{i}}{\max \left(k L_{3}^{(1)}, k L_{3}^{(2)}\right)} \quad i=1,2,3 \\
\widetilde{D_{i}} & =\frac{D_{i}}{\max \left(D_{3}^{(1)}, D_{3}^{(2)}\right)} \quad i=1,2,3
\end{aligned}
$$

where $k L_{3}^{(\ell)}$ and $D_{3}^{(\ell)}, \ell=1,2$, are the total hydrodynamic efficiency and fatigue damage after sea state $S_{3}$, respectively, for the $\ell$-th configuration. Results are shown in Fig. 14 for the representative measurement point $\left(z / d=0, \theta=0^{\circ}\right)$, since this point was shown in Section 3.3.2 to be the one which accrues the highest values of accumulated fatigue damage. Note that the sea-state optimal configuration (Cases A, B, C) renders more hydrodynamic efficiency with less accumulated fatigue damage than the annual optimal configuration (Cases D, E, F). Furthermore, it is observed that a variation on the sea-state optimal configuration has significantly more influence on the accumulated fatigue damage than on the hydrodynamic efficiency. In contrast, a variation of the incident sea state on the annual optimal configuration has significantly more influence on the hydrodynamic efficiency than on the accumulated fatigue damage.

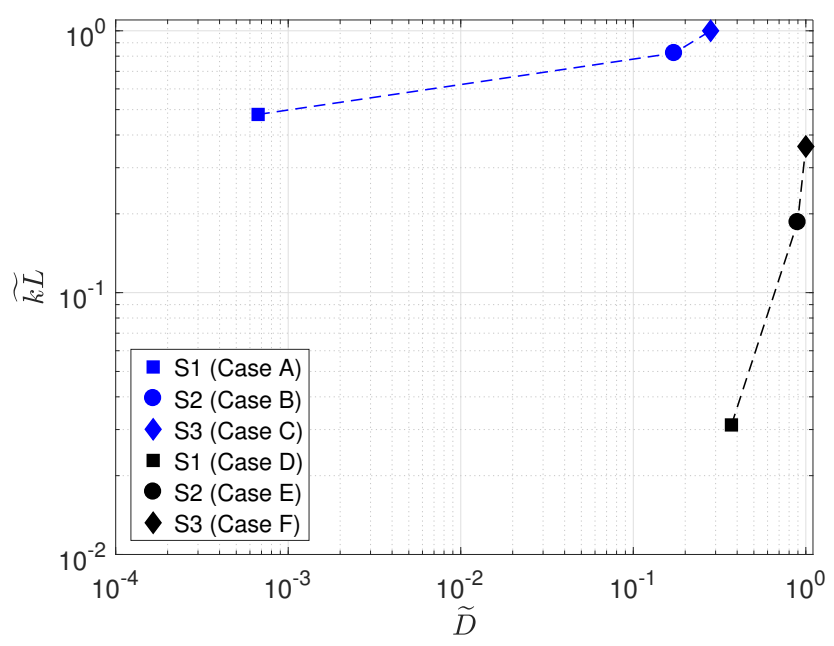

Figure 14: Dimensionless hydrodynamic efficiency against the dimensionless accumulated fatigue damage. Blue: (1) Device capable of adapting itself to the optimal configurations for sea states. Black: (2) Device optimally configured for annual values.

\section{Discussion}

The methodology in this paper is generic and can be applied to any offshore wind or marine renewable energy device. Here it has been specialised to an idealised OWC device with a series of assumptions made in order to clarify both its presentation and the obtained results. The implications of some of these modelling assumptions are discussed here. 
- The adopted spectrum to simulate the sea state corresponds to JONSWAP spectrum, one of the most commonly used model spectra for wind waves. Although it has no explicit dependence on the water depth and it was originally obtained for relative depths of the North Sea, it corresponds to a non-fully developed sea and may not represent certain situations. However, the methodology is general and can be applied at any other environmental conditions such as a totally developed sea [33].

- The simulated time for the sea state was limited to $t_{\max }=1 \mathrm{~h}$, which required a computational time of approximately $72 \mathrm{~h}$ using a $3.2 \mathrm{GHz} \mathrm{CPU}$ with $32 \mathrm{~Gb}$ RAM. However, the adopted sea states have a representative time of $3 \mathrm{~h}$, whose complete simulation would lead to a very heavy computational burden. In this sense, computational techniques such as parallel computing or surrogate modelling would be required to substantially reduce the computational cost, so that the proposed methodology can be extended to longer periods of time.

- Particular details of the OWC system such as stiffeners, supports, adaptation mechanism, etc. have been neglected in the fluid-structure interaction modelling and the FE modelling to avoid an unnecessarily complex computational problem. Depending on the type and relative sizes of such design details, the aforementioned simplification may lead to biased results. In such a case, a detailed analysis of the complete system should be carried out leading to more accurate results, but at the cost of a higher computational burden. However, this simplification does not invalidate the generic nature of the methodology presented here.

\section{Conclusions}

This paper develops a physics-based methodology to rigorously investigate the trade-off between the energetic response and the structural longevity of WEC systems in irregular waves. This methodology has been implemented in a parameterised computational model by coupling analytical and structural FE models, which enables the prediction of both the fatigue damage and the hydrodynamic efficiency of the system. The methodology has been exemplified for an idealised bottom-fixed OWC system located on the inner continental shelf in a zone of constant depth. The overall results have revealed the importance of designing OWC systems such that they can be optimally adapted to the successive sea states. In fact, this configuration not only leads to the optimal hydrodynamic efficiency, as shown by previous work of the first author, but also to less accumulated fatigue damage. In the context of the optimal control strategy for the adaptive system, both the draft and the rotational speed of the turbine have emerged as key parameters in terms of the hydrodynamic efficiency, however only the draft was preliminary revealed as the sensitive parameter in terms of cumulative fatigue damage.

A desirable extension of this research work is to substantially reduce the computational cost of this simulation methodology such that it can be efficiently and accurately applied to longer periods of time (e.g., 
a season, a year, or the lifespan). Furthermore, another immediate future research direction is to investigate the trade-off between the hydrodynamic efficiency and the structural integrity for a WECs farm, through a multi-objective optimization problem considering design decision variables such as the structural material (e.g., steel, fiber-reinforced polymers, etc.) and the arrangement of the WECs within the farm. Finally, it is emphasised that the methodology is generic and as such, it can be applied to any offshore renewable energy system.

\section{Acknowledgements}

The authors would like to acknowledge the support of the UK Centre for Marine Energy Research (UKCMER) under the SuperGen Marine programme funded by the Engineering and Physical Sciences Research Council (EPSRC), Grant EP/P008682/1. 


\section{Appendix A. Pseudo-code of the simulation tool}

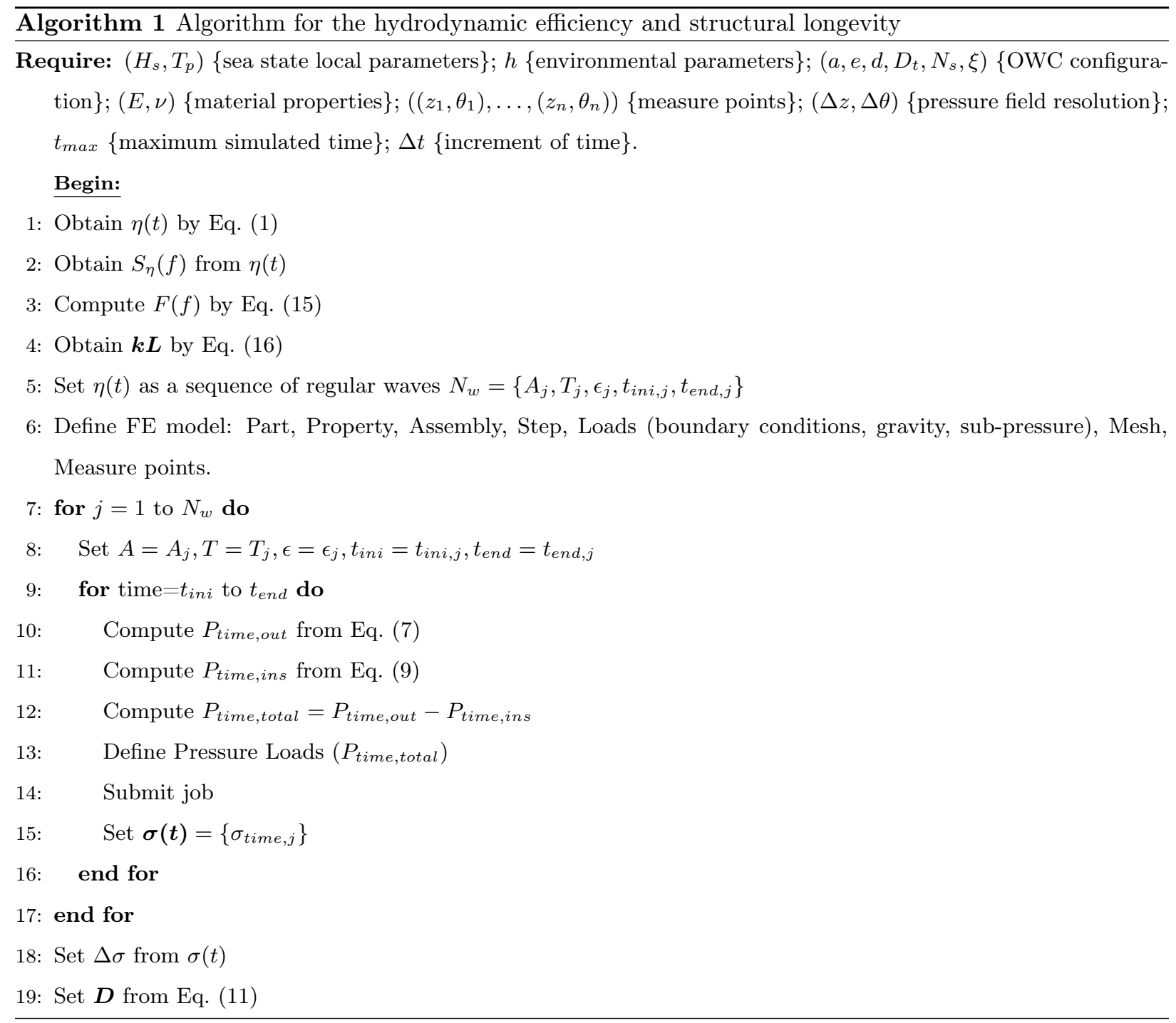

\section{Appendix B. Diffraction and radiation formulation}

The diffraction and radiation velocity potential expressions (Eq. (4)), which satisfy the boundary value problem defined by the Laplace equation, the kinematic boundary condition at the bottom, the mixed boundary condition on the free surface, and the non flux matching condition around the cylinder, are described as:

$$
\phi_{d i f}(r, \theta, z)=\left\{\begin{array}{l}
\frac{-i g A^{\prime}}{\omega} \sum_{m=0}^{N_{m}} \Gamma_{m} \sum_{l=0}^{N_{l}} B_{m l} \frac{I_{m}\left(k_{l} r\right)}{k_{l} a I_{m}^{\prime}\left(k_{l} a\right)} Z_{l}(z) \quad r<a \\
\frac{-i g A^{\prime}}{\omega} \sum_{m=0}^{N_{m}} \Gamma_{m}\left[\beta_{m} \frac{Z_{0}(z)}{Z_{0}(0)}+\sum_{l=0}^{N_{l}} B_{m l} \frac{K_{m}\left(k_{l} r\right)}{k_{l} a K_{m}^{\prime}\left(k_{l} a\right)} Z_{l}(z)\right] \quad r>a
\end{array}\right.
$$


and

$$
\phi_{\text {rad }}(r, z)=\left\{\begin{array}{l}
\frac{-i \widehat{P}}{\rho_{\omega} \omega} \sum_{l=0}^{N_{l}} D_{l} \frac{I_{0}\left(k_{l} r\right)}{k_{l} a I_{0}^{\prime}\left(k_{l} a\right)} Z_{l}(z)-\frac{i \widehat{P}}{\rho_{\omega} \omega} \quad r<a \\
\frac{-i \widehat{P}}{\rho_{\omega} \omega} \sum_{l=0}^{N_{l}} D_{l} \frac{K_{0}\left(k_{l} r\right)}{k_{l} a K_{0}^{\prime}\left(k_{l} a\right)} Z_{l}(z) \quad r>a
\end{array}\right.
$$

where $A^{\prime}=A e^{i \epsilon}$ with $\epsilon$ the phase angle, $\Gamma_{m}=\varepsilon_{m} i^{m} \cos (m \theta)$, with $\varepsilon_{0}=1, \varepsilon_{m}=2(m \geq 1)$; $\mathrm{B}_{m l}$ and $D_{l}$ are the unknown coefficients; $\beta_{m}=\left(J_{m}(k r)-H_{m}(k r) \frac{J_{m}^{\prime}(k a)}{H_{m}^{\prime}(k a)}\right) ; J_{m}$ are the Bessel functions of the first kind; $H_{m}$ are the Hankel functions; $I_{m}$ and $K_{m}$ are the modified Bessel functions; and $Z_{l}(z)$ are the depth dependent functions defined by:

$$
Z_{l}(z)=\frac{\cos k_{l}(h+z)}{\sqrt{\frac{1}{2}\left(1+\frac{\sin 2 k_{l} h}{2 k_{l} h}\right)}}, \quad l=0,1, \ldots, N_{l}
$$

In Eq. (B.3), $k_{0}=-i k$ with $k$ as the real solution of the dispersion equation, and $k_{1}, k_{2}, \ldots, k_{N_{l}}$ are the positive real solutions of:

$$
k_{l} \tan k_{l} h+\omega^{2} / g=0
$$

Since the velocity potentials inside $(r<a)$ and outside $(r>a)$ the chamber must match at their common interface, the following matching conditions are imposed to choose the unknown coefficients $B_{m l}$ and $D_{l}$ :

$$
\begin{array}{lr}
\frac{\partial \phi_{i}(r>a)}{\partial r}=\frac{\partial \phi_{i}(r<a)}{\partial r}=U(z, \theta) & r=a,-h<z<-d, 0<\theta<2 \pi \\
\phi_{i}(r>a)=\phi_{i}(r<a) & r=a,-h<z<-d, 0<\theta<2 \pi
\end{array}
$$

where $U$ is the radial velocity; and $i=d i f$, rad. 


\section{References}

[1] J. Falnes, A review of wave-energy extraction, Marine Structures 20 (4) (2007) 185-201.

[2] A. F. O. Falcão, Wave energy utilization: A review of the technologies, Renewable and Sustainable Energy Reviews 14 (3) (2010) 899-918.

[3] I. López, J. Andreu, S. Ceballos, I. M. de Alegría, I. Kortabarria, Review of wave energy technologies and the necessary power-equipment, Renewable and Sustainable Energy Reviews 27 (2013) 413-434.

[4] E. Ozkop, I. H. Altas, Control, power and electrical components in wave energy conversion systems: A review of the technologies, Renewable and Sustainable Energy Reviews 67 (2017) 106-115.

[5] A. Clément, P. McCullen, A. F. O. Falcão, A. Fiorentino, F. Gardner, K. Hammarlund, G. Lemonis, T. Lewis, K. Nielsen, S. Petroncini, et al., Wave energy in Europe: current status and perspectives, Renewable and Sustainable Energy Reviews 6 (5) (2002) 405-431.

[6] R. P. F. Gomes, J. C. C. Henriques, L. M. C. Gato, A. F. O. Falcão, Hydrodynamic optimization of an axisymmetric floating oscillating water column for wave energy conversion, Renewable Energy 44 (2012) 328-39.

[7] T. Vyzikas, S. Deshoulières, M. Barton, O. Giroux, D. Greaves, D. Simmonds, Experimental investigation of different geometries of fixed oscillating water column devices, Renewable Energy 104 (2017) 248-258.

[8] F. R. Torres, P. R. Teixeira, E. Didier, A methodology to determine the optimal size of a wells turbine in an oscillating water column device by using coupled hydro-aerodynamic models, Renewable Energy 121 (2018) 9-18.

[9] M. Shadman, S. F. Estefen, C. A. Rodriguez, I. C. Nogueira, A geometrical optimization method applied to a heaving point absorber wave energy converter, Renewable Energy 115 (2018) 533-546.

[10] A. F. O. Falcão, R. J. A. Rodrigues, Stochastic modelling of OWC wave power plant performance, Applied Ocean Research 24 (2) (2002) 59-71.

[11] A. F. O. Falcão, Phase control through load control of oscillating-body wave energy converters with hydraulic PTO system, Ocean Engineering 35 (3-4) (2008) 358-366.

[12] A. F. O. Falcão, J. C. Henriques, J. J. Cândido, Dynamics and optimization of the OWC spar buoy wave energy converter, Renewable Energy 48 (2012) 369-381.

[13] M. L. Jalón, A. Baquerizo, M. Losada, Optimization at different time scales for the design and management of an oscillating water column system, Energy 95 (2016) 110-123.

[14] N. Tom, M. Lawson, Y.-H. Yu, A. Wright, Spectral modeling of an oscillating surge wave energy converter with control surfaces, Applied Ocean Research 56 (2016) 143-156.

[15] N. M. Tom, Y.-H. Yu, A. D. Wright, M. J. Lawson, Pseudo-spectral control of a novel oscillating surge wave energy converter in regular waves for power optimization including load reduction, Ocean Engineering 137 (2017) 352-366.

[16] N. M. Tom, F. Madhi, R. W. Yeung, Power-to-load balancing for heaving asymmetric wave-energy converters with nonideal power take-off, Renewable Energy 131 (2019) 1208-1225.

[17] G. Van Rossum, F. L. Drake Jr, Python tutorial, Centrum voor Wiskunde en Informatica Amsterdam, The Netherlands, 1995.

[18] Hibbitt, Karlsson, Sorensen, ABAQUS/standard User's Manual, Vol. 1, Hibbitt, Karlsson \& Sorensen, 2001.

[19] S. K. Chakrabarti, Hydrodynamics of offshore structures, WIT press, 1987.

[20] K. Hasselmann, T. Barnett, E. Bouws, H. Carlson, D. Cartwright, K. Enke, J. Ewing, H. Gienapp, D. Hasselmann, P. Kruseman, et al., Measurements of wind-wave growth and swell decay during the Joint North Sea Wave Project (JONSWAP), Ergänzungsheft (1973), 8-12.

[21] D. N. Veritas, Recommended practice DNV-RP-C205 Environmental conditions and environmental loads, RP-C205, 2010.

[22] H. Martins-Rivas, C. C. Mei, Wave power extraction from an oscillating water column at the tip of a breakwater, Journal of Fluid Mechanics 626 (2009) 395-414. 
[23] C. Garrett, Bottomless harbours, Journal of Fluid Mechanics 43 (3) (1970) $433-449$.

[24] D. V. Evans, R. Porter, Efficient calculation of hydrodynamic properties of OWC-type devices, Journal of Offshore Mechanics and Arctic Engineering 119 (4) (1997) 210-218.

[25] R. G. Dean, R. A. Dalrymple, Water wave mechanics for engineers and scientists, Vol. 2, World Scientific Publishing Company, 1991.

[26] J. Schijve, Fatigue of structures and materials, Springer Science \& Business Media, 2001.

[27] M. S. Longuet-Higgins, On the statistical distribution of the height of sea waves, Journal of Marine Research 11 (1952) $245-266$.

[28] M. L. Jalón, A. Lira-Loarca, A. Baquerizo, M. Á. Losada, An analytical model for oblique wave interaction with a partially reflective harbor structure, Coastal Engineering 143 (2019) 38-49.

[29] ABAQUS 6.9, Analysis User's Manual, Vol. IV:Elements, 2009.

[30] P. A. Brodtkorb, P. Johannesson, G. Lindgren, I. Rychlik, J. Ryden, E. Sjö, et al., WAFO-a Matlab toolbox for analysis of random waves and loads, in: The Tenth International Offshore and Polar Engineering Conference, International Society of Offshore and Polar Engineers, 2000.

[31] N. T. Kottegoda, R. Rosso, Applied statistics for civil and environmental engineers, Blackwell Malden, MA, 2008.

[32] D. N. Veritas, Fatigue design of offshore steel structures, DNV Recommended Practice DNV-RP-C203, 2010.

[33] W. J. Pierson Jr, L. Moskowitz, A proposed spectral form for fully developed wind seas based on the similarity theory of SA Kitaigorodskii, Journal of Geophysical Research 69 (24) (1964) 5181-5190. 